\title{
Analysis of Homogeneous Broadening in n-type doped Ge layers on Si for laser application
}

\author{
S. A. Srinivasan ${ }^{1,2,{ }^{*}, \text { C. } \text { Porret }^{1}, \text { M. Pantouvaki }}{ }^{1}$, Y. Shimura ${ }^{1,3}$, P. Geiregat ${ }^{2,4}$, R. Loo ${ }^{1}$, J. Van Campenhout ${ }^{1}$, D. \\ Van Thourhout ${ }^{2}$ \\ ${ }^{1}$ Imec, Kapeldreef 75, Heverlee B-3001, Belgium \\ ${ }^{2}$ Photonics Research Group, Dept. of Information Technology, Ghent University-imec \& Center for Nano- and Biophotonics, Ghent \\ University, Technologiepark-Zwijnaarde 15 iGent, 9052 Ghent, Belgium \\ ${ }^{3}$ Current address: Shizuoka University, Hamamatsu 432-8001, Japan \\ ${ }^{4}$ Physics and Chemistry of Nanostructures, Ghent University, 9000 Ghent, Belgium \\ "Author e-mail address: ashwyn.srinivasan@imec.be
}

\begin{abstract}
The homogeneous broadening in Phosphorus doped Ge layers is characterized using photoluminescence spectroscopy and absorption measurements. A broadening parameter $\Gamma_{\text {Hом }}=45 \mathrm{meV}$ due to carrier scattering effects was extracted leading to an estimated increase in threshold current density for Ge lasers by a factor $>4$.
\end{abstract}

Index Terms - Laser; Homogeneous broadening; Silicon photonics

\section{INTRODUCTION}

Silicon photonics $(\mathrm{SiPh})$ based optical interconnects are widely considered for short-reach interconnect applications where their electrical counterparts face the bandwidth-power density bottleneck [1]. An important component of every optical transceiver is the laser source and Germanium $(\mathrm{Ge})$ is considered to be a promising material for realizing it in the scope of $\mathrm{SiPh}$. Ge is intrinsically an indirect band gap material but can be made pseudo direct band gap by doping it with Phosphorus (P) $[2,3]$. However, doping Ge with $>2 \times 10^{19} \mathrm{~cm}^{-3}$ $\mathrm{P}$ atoms reduces the Shockley-Reed-Hall recombination lifetime of carriers and introduces additional carrier scattering effects due to phonons and impurities in the lattice [4]. These carrier scattering effects result in homogeneous broadening of the gain spectrum and increases the threshold current density of the laser. In this work, we extract the broadening parameter ( $\left.\Gamma_{\text {ном}}\right)$ of $\mathrm{P}$ doped $\mathrm{Ge}$ layers using photoluminescence spectroscopy (PL) and absorption measurements. A modified Joint Density of State (JDOS) model that considers many-body effects such as homogeneous broadening, the Urbach tail and dopant induced band-gap narrowing is used in this study.

\section{II.MODELING HOMOGENEOUS BROADENING}

The JDOS model is a commonly used technique to calculate the absorption, spontaneous emission and gain spectra of a semiconductor. However, one typically assumes an ideal quadratic JDOS together with recombination or carrier generation physics that obeys conservation of momentum and follows the k-selection rule. Deviations from this ideal behaviour are the result of many-body-effects due to phonons, carrier, impurities or defects. These deviations can be captured with the addition of an Urbach tail (an exponential tail below the band-edge), including homogeneous broadening effects (through convolution of the absorption and spontaneous emission spectra with a Lorentzian line shape function) and dopant induced band-gap narrowing effects (BGN) as proposed in $[5,6]$. Such a modified JDOS model is used here to model the measured photoluminescence and absorption spectra of $\mathrm{P}$ doped Ge samples. The full width at half maximum (FWHM) of the Lorentzian line shape function used to model the homogeneous broadening effects is represented here as the broadening parameter- $\Gamma_{\text {ном. }}$.

\section{SAMPLE AND EXPERIMENT DETAILS}

P-doped Ge layers of $0.2 \mu \mathrm{m}$ were grown on $0.2 \mu \mathrm{m}$ thick Ge buffer layers deposited on $\mathrm{Si}(001)$ substrates by a RPCVD process in a $300 \mathrm{~mm}$ Intrepid ${ }^{\mathrm{TM}}$ XP system from ASM [7], with $\mathrm{Ge}_{2} \mathrm{H}_{6}$ and $\mathrm{PH}_{3}$ as the precursors. Rapid thermal annealing at $700^{\circ} \mathrm{C}$ for $30 \mathrm{sec}$ in $\mathrm{N}_{2}$ allows diffusion of P-atoms into the underlying undoped $\mathrm{Ge}$ buffer, resulting in uniformly doped 0.4 $\mu \mathrm{m} \mathrm{Ge}$ layers on $\mathrm{Si}$, and also improves the crystallinity of the layer by reducing point defects. A summary of all samples investigated in this work is shown in Table 1. Their doping level is similar to that of the electrically pumped $\mathrm{Ge}$ laser demonstrated in $[8,9]$. An undoped layer of $0.6 \mu \mathrm{m}$ Ge was used as a reference.

Table 1: Summary of sample details investigated in this paper.

\begin{tabular}{|c|c|c|}
\hline Sample detail & Chem. P conc. & Active P conc. \\
\hline $0.6 \mu \mathrm{m} \mathrm{Ge}$ buffer (reference) & - & - \\
\hline $320^{\circ} \mathrm{C} \mathrm{ppPH} / \mathrm{ppGe}_{2} \mathrm{H}_{6}=0.006$ & $2.8 \times 10^{19} \mathrm{~cm}^{-3}$ & $2.65 \times 10^{19} \mathrm{~cm}^{-3}$ \\
\hline $320^{\circ} \mathrm{C} \mathrm{ppPH}_{3} / \mathrm{ppGe}_{2} \mathrm{H}_{6}=0.02$ & $5.3 \times 10^{19} \mathrm{~cm}^{-3}$ & $4.5 \times 10^{19} \mathrm{~cm}^{-3}$ \\
\hline $425^{\circ} \mathrm{C} \mathrm{ppPH}_{3} / \mathrm{ppGe}_{2} \mathrm{H}_{6}=0.02$ & $5.2 \times 10^{19} \mathrm{~cm}^{-3}$ & $4.5 \times 10^{19} \mathrm{~cm}^{-3}$ \\
\hline
\end{tabular}

Photoluminescence (PL) and absorption measurements were used to characterize the samples. The PL measurement was performed by exciting the sample with pump intensities in the range of $0.03-0.2 \mathrm{MW} / \mathrm{cm}^{2}$ at a wavelength of $532 \mathrm{~nm}$. The PL spectra were collected using an extended-InGaAs detector covering the range 1.3-2.2 $\mu \mathrm{m}$. Absorption measurements were performed using a UV-VIS-IR Spectrophotometer. 

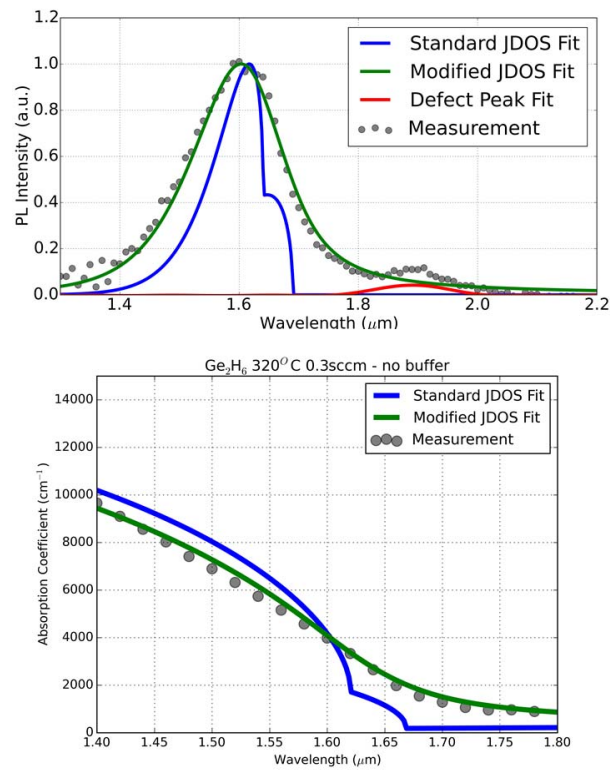

Fig. 1. PL and absorption spectra of the sample with growth condition $-320^{\circ} \mathrm{C} \mathrm{ppPH} / \mathrm{ppGe}_{2} \mathrm{H}_{6}=0.006$. The PL spectra was obtained with a pump intensity of $0.08 \mathrm{MW} / \mathrm{cm}^{2}$. The modified JDOS model was used to extract the broadening parameter (Гном), Urbach tail (Eo) and dopant induced band-gap narrowing (BGN).

Table 2: Summary of extracted broadening parameter $\left(\Gamma_{\text {ном }}\right)$, Urbach tail $\left(\mathrm{E}_{\mathrm{O}}\right)$ and dopant induced band gap narrowing (BGN) using the modified JDOS model.

\begin{tabular}{|c|c|c|c|}
\hline Sample detail & $\begin{array}{c}\mathrm{E}_{\mathrm{O}} \\
(\mathrm{meV})\end{array}$ & $\begin{array}{c}\Gamma_{\mathrm{HOM}} \\
(\mathrm{meV})\end{array}$ & $\begin{array}{c}\text { BGN } \\
(\mathrm{meV})\end{array}$ \\
\hline $0.6 \mu \mathrm{m} \mathrm{Ge} \mathrm{buffer} \mathrm{(reference)}$ & $8 \pm 1.5$ & $3 \pm 2.5$ & 0 \\
\hline $320^{\circ} \mathrm{C} \mathrm{ppPH}_{3} / \mathrm{ppGe}_{2} \mathrm{H}_{6}=0.006$ & $5 \pm 1.5$ & $45 \pm 2.5$ & 25 \\
\hline $320^{\circ} \mathrm{C} \mathrm{ppPH}_{3} / \mathrm{ppGe}_{2} \mathrm{H}_{6}=0.02$ & $5 \pm 1.5$ & $45 \pm 2.5$ & 37.5 \\
\hline $425^{\circ} \mathrm{C} \mathrm{ppPH}_{3} / \mathrm{ppGe}_{2} \mathrm{H}_{6}=0.02$ & $5 \pm 1.5$ & $50 \pm 2.5$ & 37.5 \\
\hline
\end{tabular}

\section{RESULTS AND DISCUSSION}

Fig. 1. shows the PL measurement and absorption coefficient of the sample where the P-doped Ge layer was grown at $320^{\circ} \mathrm{C}$ with $\mathrm{ppPH}_{3} / \mathrm{ppGe}_{2} \mathrm{H}_{6}=0.006$. The $\mathrm{PL}$ spectrum shown in the figure was obtained for a pumping intensity of 0.08 $\mathrm{MW} / \mathrm{cm}^{2}$. The spontaneous emission associated with the direct $\Gamma$-valley results in a PL emission peak at $1.62 \mu \mathrm{m}$. The peak at $1.9 \mu \mathrm{m}$, which is not present in the PL spectrum from undoped $\mathrm{Ge}$, is linked to defects present in the doped layers due to introduction of P dopants. In order to model the PL emission from the $\Gamma$ valley, the carrier concentration in the layers was estimated by solving the carrier-continuity equation for each pump intensity assuming a cylindrical distribution of photoexcited carriers in the Ge layer. A Shockley-Reed-Hall recombination lifetime of $4 \mathrm{~ns}$ and $0.4 \mathrm{~ns}$ was considered for undoped and doped Ge respectively [10,11]. Taking these operating condition into account, we model an ideal PL spectra governed by the k-selection rule, without Urbach tail. However, this ideal spectrum does not well represent the measurements as is clear from Fig. 1. To overcome this problem, we included many-body effects and swept $\Gamma_{\text {Hом }}, E_{0}$ and BGN to reproduce the PL and absorption spectra obtained experimentally. An example is shown in Fig. 1 where $\Gamma_{\mathrm{HOM}}=45 \mathrm{meV}, \mathrm{E}_{\mathrm{O}}=5 \mathrm{meV}$ and $\mathrm{BGN}=25 \mathrm{meV}$ were used in the fit. For all doped Ge layers we found $\Gamma_{\text {Ном }} \geq 45 \mathrm{meV}$ compared to $\Gamma_{\mathrm{HOM}}=3 \mathrm{meV}$ for undoped Ge layers (see Table 2). We believe the high broadening parameter for doped $\mathrm{Ge}$ arises from carrier-carrier scattering, carrier-neutral impurity scattering, carrier-ionized impurity scattering, or carrier- acoustical/optical phonon scattering effects. These additional scattering effects generate broadening in the gain spectrum and lead to a substantial increase in the calculated threshold current density as shown in Fig 2 and in $[5,6]$.

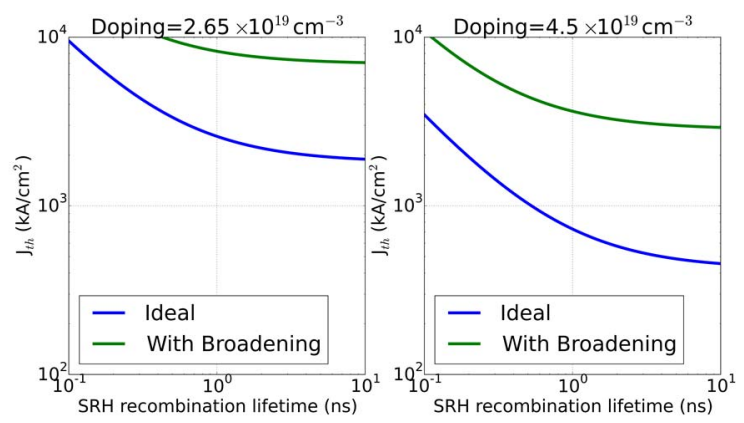

Fig. 2. Calculated threshold current density as a function of SRH recombination lifetime for n-type doped Ge layers. Simulation includes a target modal loss of $200 \mathrm{~cm}^{-1}$ and $\Gamma_{\mathrm{HOM}}=45 \mathrm{meV}, \mathrm{E}_{\mathrm{O}}=5$ meV for curves named "With Broadening".

\section{V.CONCLUSION}

In this work, we investigated homogeneous broadening due to carrier scattering effects in Phosphorus doped Ge layers grown on Si . By fitting results from photoluminescence and absorption spectroscopy with a modified JDOS model we could extract the broadening parameter $\left(\Gamma_{\mathrm{HOM}}\right)$. For uniformly doped Ge layers up to a doping level of $4.5 \times 10^{19} \mathrm{~cm}^{-3}, \Gamma_{\text {Ном }} \geq 45 \mathrm{meV}$ was extracted as compared to $\Gamma_{\mathrm{HOM}}=3 \mathrm{meV}$ for undoped $\mathrm{Ge}$ layers. From these findings we estimate that the threshold current density of a Ge laser with active $\mathrm{P}$ doping is increased by a factor of 4 to 8 compared with a model where this broadening is not taken into account.

\section{ACKNOWLEDGEMENTS}

Y. Shimura acknowledges the Research Foundation of Flanders (FWO) for granting him a fellowship within the Pegasus Marie Curie Program. This work was supported by imec's industry-affiliation program on Optical I/O. Air Liquide Advanced Materials is acknowledged for providing $\mathrm{Ge}_{2} \mathrm{H}_{6}$.

\section{REFERENCES}

[1] D.A.B. Miller, et al., Proc. IEEE, vol. 97, no. 7, pp. 1166-1185, Jul. 2009.

[2] S. A. Srinivasan, et al., J. Lightwave Technol., 34, 419-424, 2016.

[3] S. Wirths, et al., Nature Photonics, 9(2), 88-92, 2015.

[4] L. A. Coldren, et al., Wiley, Second edition, 2012, Chapter 4.

[5] A. Garib, et al., Advanced Optical Materials, vol. 3, no. 3, 2015.

[6] M. Prost, et al., Journal of Applied Physics, 125704, 125704-1-7, 2015.

[7] Y. Shimura, et al., Thin Solid Films, 602, 56-69, 2016.

[8] R. E. Camacho-Aguilera, et al., Optics Express 20, 11316-11320, 2012.

[9] R. Koerner, et al., Optics Express 23, 14815-14822, 2015.

[10] R. Geiger, et al., Applied Physics Letters, 104(6), 062106, 2014.

[11] S. A. Srinivasan, et al., Applied Physics Letters, 108(21), 211101, 2016. 